\title{
The prevalence of urinary tract infection in children with severe acute malnutrition: a narrative review
}

\section{Samuel N Uwaezuoke \\ Department of Pediatrics, Pediatric Nephrology Firm, University of Nigeria Teaching Hospital, Ituku- Ozalla, Enugu, Nigeria}

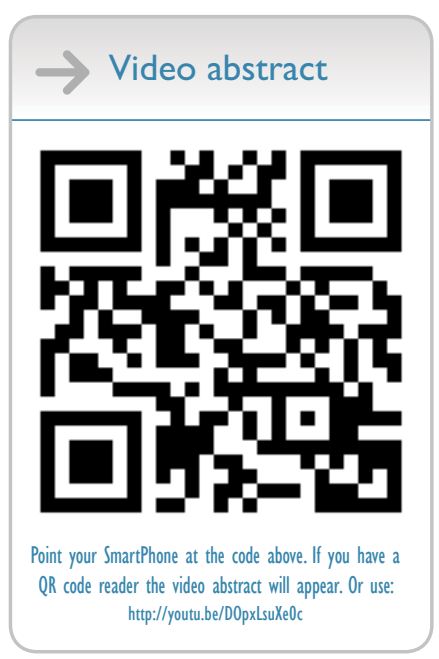

Correspondence: Samuel N Uwaezuoke Department of Pediatrics, Pediatric Nephrology Firm, University of Nigeria Teaching Hospital, Ituku-Ozalla, Enugu 40000I, Nigeria

Tel +2348033248108

Email snuwaezuoke@yahoo.com
This article was published in the following Dove Press journal:

Pediatric Health, Medicine and Therapeutics

4 October 2016

Number of times this article has been viewed

Abstract: This article aims to review the current evidence which shows that the prevalence of urinary tract infection (UTI) has been increasing in children with severe acute malnutrition (SAM). UTI remains one of the most common causes of febrile illness in pediatric practice. Most studies conducted among hospitalized children with complicated SAM have reported high prevalence rates of UTI. Clearly, the knowledge of baseline risk of UTI can help clinicians to make informed diagnostic and therapeutic decisions in these children. From the global reports reviewed in this article, UTI prevalence rates range from as low as $6 \%$ to as high as $37 \%$ in developing countries, while the most common bacterial isolates from urine cultures are Gramnegative coliform organisms such as Escherichia coli and Klebsiella species. These findings form the basis for the current diagnostic and therapeutic guidelines for clinicians managing children with complicated SAM. With the reported high prevalence of UTI among these children and concerns over antibiotic resistance, more extensive data are required using standardized microbiological methods. Thus, the assessment of the performance of urine dipsticks and microscopy against the gold standard urine culture is an important step toward strengthening the evidence for the therapeutic guidelines for UTI in children with SAM.

Keywords: protein energy malnutrition, bacterial infection, urinary tract, therapeutic guidelines

\section{Introduction}

Urinary tract infection (UTI) remains one of the most common causes of febrile illness in pediatric practice. ${ }^{1-4}$ In developing countries, it ranks next to gastrointestinal and respiratory tract infections as the third most common bacterial infection in children. ${ }^{5}$

A number of host factors predispose children to UTI; these include obstructive uropathy, urolithiasis, incomplete emptying of the bladder with residual urine, noncircumcision in boys, female sex after infancy, and constipation. ${ }^{6}$ Nevertheless, there are some host defense mechanisms, namely, the intrinsic defense of the bladder epithelial cells, secretory immunoglobulin A ( $\operatorname{IgA})$ in urine, and blood group antigens in secretions, which block bacterial adhesion to the lining of the urinary tract. ${ }^{5}$

UTI is more common in malnourished children than in their well-nourished counterparts, and the risk of UTI increases with the severity of malnutrition. ${ }^{7}$ Severe acute malnutrition (SAM) is associated with immune deficiency, which expectedly renders affected children more vulnerable to severe infections. ${ }^{8-10}$ As defined in the joint statement by World Health Organization (WHO) and United Nations Children's Emergency Fund, SAM consists of two entities: severe wasting and nutritional edema. ${ }^{11-13}$ Severe wasting (marasmus) is defined as weight-for-height below -3 standard 
deviations (or $Z$-scores) or middle upper arm circumference $<11.5 \mathrm{~cm}^{11-13}$ Nutritional edema (kwashiorkor) is defined as bilateral pitting edema independent of weight-for-height.

Most studies conducted in developing countries among hospitalized children with complicated SAM (hypoglycemia, hypothermia, lethargy, or other signs of illness) have reported high prevalence rates of UTI. ${ }^{14-21}$ Clearly, the knowledge of the baseline risk of UTI can help clinicians to make informed diagnostic and therapeutic decisions in these children.

Thus, although there have been no randomized trials with regard to the diagnostic strategies or different treatments for UTI in children with SAM, some authors recommend dipstick urinalysis/urine microscopy and antimicrobial treatment for all children admitted with complicated SAM given the high UTI prevalence rates. ${ }^{22}$

This article aims to review the current evidence about the increased UTI prevalence in children with SAM and the basis for the suggested treatment guidelines in affected children.

Using appropriate search terms, information was gathered from Google, PubMed, and Medline databases, as well as from relevant textbooks and web links. Recent studies on UTI prevalence in children with SAM were appraised to present an overview of their findings.

\section{Studies on UTI prevalence in children with SAM: an appraisal}

Several studies have been conducted in many developing countries in Africa, ${ }^{14-21}$ Asia, ${ }^{7}$ and the Middle East, ${ }^{23}$ which have reported varying prevalence rates of UTI in children with SAM (Table 1). Notably, there are no regional disparities with respect to the implicated bacterial organisms; the most common isolated organisms remain Gram-negative bacilli with minor differences in the species reported across the countries and regions.

\section{The immune system and UTI risk}

Generally, the effects of malnutrition on the immune system include the following: reduced cell-mediated immunity; diminished IgA response; reduced levels of complements; decreased efficacy of phagocytes; atrophy of lymph glands, tonsils, and thymus; as well as reduced inflammatory response and migration of white blood cells to areas of tissue damage. Since one of the defense mechanisms against UTI is the elaboration of secretory IgA in urine, it is not surprising that with the diminished IgA response seen in malnutrition, children with SAM are susceptible to UTI. The role of secretory IgA in UTI episodes has been well documented in many reports. ${ }^{24-27}$

Some investigators examined the changes in secretory $\operatorname{IgA}(\operatorname{sg} \mathrm{A}), \operatorname{Ig} \mathrm{A}$, and free secretory component during the first year of life in relation to age, sex, and infants' feeding practice; when healthy children were compared with those with acute and recurrent UTI, acute UTI significantly resulted in raised $\operatorname{sig} \mathrm{A}$, IgA, and free secretory component compared with controls. ${ }^{24}$ This finding was corroborated by other authors who observed that the elevation of $\operatorname{sigA}$ in urine not only correlated with UTI in children but that SIgA also seemed to be directed to the infective agent and could thus be used to identify the type of infection. ${ }^{25}$ Other workers noted that female children with symptomatic UTI, who had normal urinary tracts, had significantly higher sIgA excretion rates than in either controls or girls without symptoms at the time of study; ${ }^{26}$ urinary sIgA excretion rates were particularly highest in children with symptomatic UTI who had an

Table I UTI prevalence in children with complicated SAM: the situation in some developing countries

\begin{tabular}{|c|c|c|c|}
\hline Study & Country (continent) & UTI prevalence & Most common bacterial isolates \\
\hline \multirow[t]{2}{*}{ Rabasa and Shattima ${ }^{14 a}$} & Nigeria (Africa) & $11 \%$ & Gram-negative bacilli especially \\
\hline & & & Escherichia coli \\
\hline Page et $\mathrm{al}^{15 \mathrm{a}}$ & Niger (Africa) & $16 \%$ & E. coli \\
\hline Okomo et $\mathrm{al}^{35 \mathrm{a}}$ & The Gambia (Africa) & $16.5 \%$ & Klebsiella sp. \\
\hline Shimeles and Lulseged ${ }^{20 a}$ & Ethiopia (Africa) & $37 \%$ & E. coli and Klebsiella sp. \\
\hline Thuo et $\mathrm{al}^{2 \mathrm{la}}$ & Kenya (Africa) & $6 \%$ (culture-proven), 24\% (positive dipstick) & Gram-negative enteric bacilli \\
\hline Ahmed et $\mathrm{al}^{34 \mathrm{a}}$ & Tanzania (Africa) & $20.3 \%$ & Coliforms \\
\hline Kala and Jacobs ${ }^{16 a}$ & South Africa (Africa) & $34.7 \%$ & E. coli and Klebsiella sp. \\
\hline Berkowitz $^{18 a}$ & South Africa (Africa) & $31 \%$ & E. coli \\
\hline Caksen et $\mathrm{al}^{23 \mathrm{a}}$ & Turkey (Middle East) & $30 \%$ & E. coli \\
\hline Brown et $\mathrm{al}^{36 \mathrm{a}}$ & Bangladesh (Asia) & $30 \%$ & E. coli \\
\hline Bagga et $\mathrm{al}^{7 \mathrm{~b}}$ & India (Asia) & $15.2 \%$ & Gram-negative bacilli \\
\hline Banapurmath and Jayamony ${ }^{33 b}$ & India (Asia) & $8 \%$ & Gram-negative bacilli \\
\hline
\end{tabular}

Notes: aProspective studies; ${ }^{b}$ prospective case-control studies.

Abbreviations: UTI, urinary tract infection; SAM, severe acute malnutrition. 
abnormal urinary tract. Furthermore, it was concluded from another study that low urinary SIgA may also represent an important predisposing factor to recurrent UTI. ${ }^{27}$

The effect of malnutrition on IgA has been established albeit in animal studies. ${ }^{28-30}$ In one of the studies, the results suggested that protein deprivation resulted in a reversible reduction in the IgA response to antigens. ${ }^{28}$ Similarly, other researchers specifically demonstrated that the protein deprivation in mice is accompanied by the stimulation of Lyt-2+ suppressor T cells which suppress IgA response after oral antigenic exposure. ${ }^{29}$ In addition, the findings from a related study showed that dietary protein played a significant, site-specific role in the developmental expression of the secretory immune system, as severe protein malnutrition dramatically suppressed this immune component in the rat models. ${ }^{30}$

Although the pattern of UTI etiologic agents is similar in malnourished and non-malnourished children, transferrin levels are generally reduced in SAM, leading to free, unbound iron in circulation. This is thought to provide a milieu for Gram-negative organisms to thrive, resulting in Gram-negative sepsis and subsequently UTI through the hematogenous route.

\section{UTI prevalence rates and the isolated organisms}

In the West African subregion, a hospital-based prospective study in Nigeria documented a prevalence rate of $\sim 11 \%$. ${ }^{14}$ The authors investigated 194 children with severe proteinenergy malnutrition, leading to UTI, and noted no difference between the sexes. The absence of sex difference in the study could be explained by the predominant age bracket of the recruited patients. Given the known epidemiological pattern of UTI, the prevalence generally appears to be equal for both sexes during infancy, with male predominance in the neonatal period and female predominance during early childhood and the period of toilet training. Furthermore, Gram-negative organisms with preponderance of Escherichia coli were the most common isolates: a finding which also underscores the fact that majority of the subjects were possibly not yet toilet trained. In the neighboring Niger Republic, other workers documented a prevalence of $16 \%$ in 300 hospitalized children with complicated SAM. ${ }^{15}$ E. coli represented more than $75 \%$ of the microorganisms isolated, followed by Klebsiella pneumoniae, Proteus mirabilis, and E. faecium. UTI were more frequent in children under 1 year of age and there was no significant association with sex. The urinary dipstick was positive for leukocytes and/or nitrites in half of the children with culture-proven UTI.

In a similar study in South Africa, other investigators evaluated 75 children with malnutrition by urine culture on suprapubic specimens. ${ }^{16}$ Imaging studies such as renal ultrasound, intravenous urography, and micturating cystourethrography were also conducted, and the results did not reveal anatomic abnormalities and reflux, thus excluding confounders of UTI risk. The prevalence rate of UTI was 34.7\% with a male preponderance. The overall prevalence of UTI in children with kwashiorkor and marasmic-kwashiorkor was $42 \%$. This study gives a comparatively high prevalence rate. It is possible that a confounding factor such as absence of circumcision was not considered, since majority of the subjects who had UTI were reportedly male children. Again, the most commonly cultured organism was $E$. coli as was noted in the Nigerian study. ${ }^{14}$ Other workers in a rural hospital in South Africa reported a UTI prevalence of $26.1 \%$ after investigating 134 children, under the age of 5 years, with all grades of malnutrition. ${ }^{17}$ In a prospective study, one researcher in the same country reported a UTI prevalence of $31 \%$ in 68 Negroid children admitted to the hospital with kwashiorkor, marasmus, or marasmic-kwashiorkor. ${ }^{18}$ The UTIs that were diagnosed using suprapubic urine specimens were caused by E. coli. When compared to the study conducted in Nigeria, the prevalence rates in these South African studies appear to be relatively high. Perhaps, the malnourished children in these studies ${ }^{18,19}$ also had other comorbid immunodeficiency states such as retroviral infection; its seroprevalence being notably high in South Africa. ${ }^{19}$ Nevertheless, some workers in South Africa have documented that HIV/AIDS per se showed no significant impact on the presentation of UTI in children. ${ }^{31}$ In the study in which they compared HIV-positive and HIV-negative children with UTI, they noted that the clinical presentation, etiological agents, response to therapy, and renal function were similar in both groups. ${ }^{31}$ In addition, the rate of male circumcision - a confounding variable - is comparatively low in the country. In an Indian study, which assessed the pattern of periurethral bacterial flora in uncircumcised boys and evaluated the effect of circumcision on alteration of periurethral uropathogenic bacterial flora, the investigators have provided circumstantial evidence supporting the idea that early circumcision may be beneficial for the prevention of UTI in male children. ${ }^{32}$

Elsewhere in Ethiopia, a prospective study on the clinical profile and pattern of infection in 90 children with severe protein-energy malnutrition reported a high UTI prevalence of 
$37 \%{ }^{20}$ The study group consisted of 44 (49\%) children with marasmus, 29 (32\%) with marasmic-kwashiorkor, and 17 (19\%) with kwashiorkor, with their ages ranging from 4 to 60 months. The isolated bacterial pathogens were predominantly Gram-negative enteric organisms. Again, it is possible that confounders such as sex and circumcision could have accounted for this high prevalence figure. In addition, the age bracket of the study population was within the pretoilet training and toilettraining period, a phase that contributes to UTI risk in children.

In another hospital-based study in Turkey (Middle East), 103 children with malnutrition were investigated prospectively for UTI. ${ }^{23}$ The authors reported a UTI prevalence rate of $30 \%$. Similar to the previous reports, the most common isolated microorganism from urine cultures was $E$. coli. The major limitation in all these studies ${ }^{14-18,20}$ was the methodology that was adopted as it failed to include nonmalnourished controls. It would have been a more acceptable study design to validate the observation that children with SAM have increased risk of UTI.

Nevertheless, a prospective study in India (Asia) examined the incidence of bacteriuria in malnourished children between 6 months and 5 years of age using a better methodology. ${ }^{7}$ The workers included for each malnourished patient, a nonmalnourished control matched for age, sex, and presence of fever and diarrhea. Significant bacteriuria was noted in $17(15.2 \%)$ malnourished and two (1.8\%) nonmalnourished controls $(P<0.01)$. The difference between the incidence of bacteriuria in febrile malnourished $(28.6 \%)$ and nonmalnourished subjects $(5.7 \%)$ was considered to be statistically significant. The authors therefore concluded that malnourished children, particularly those with fever, were at risk of UTI. ${ }^{7}$ In another study in India using the same methodology, the investigators enrolled 88 severely malnourished preschool children to determine the prevalence of UTI. ${ }^{33}$ Fifty-three well-nourished preschool children served as controls. UTI was reported in $8 \%$ of the malnourished children.

In Tanzania (East Africa), a group of researchers studied 402 malnourished children under the age of 5 years, predominantly of male sex, and reported that $20.3 \%$ had significant bacteriuria consisting of Gram-negative enteric bacteria, ${ }^{34}$ E. coli and $K$. pneumoniae being the predominantly isolated bacteria. In another East African country (Kenya), a 2-year prospective hospital-based study conducted on 498 malnourished children showed a positive dipstick test (leukocyte esterase and nitrite) for UTI in $24 \%$ of the cases; however, culture-proven UTI was detected in $6 \%$ of the children. ${ }^{21}$ It is remarkable to note that all the bacterial isolates in the study were coliforms.
In one prospective hospital-based study in the Gambia, among 140 children admitted with complicated SAM, the authors reported the prevalence of bacteriuria as $16.5 \% .{ }^{35}$ The predominant urinary pathogens were E. coli and Klebsiella species. Finally, in another Asian country (Bangladesh), a systematic evaluation of 100 children admitted to hospital for severe protein-calorie malnutrition was conducted for the presence of infections $;{ }^{36}$ the workers reported a UTI prevalence rate of $30 \%$.

In summary, there appears to be unanimity in the findings of these studies on the predominance of Gram-negative organisms as etiologic agents but with little or no regional differences.

\section{Severity/grades of malnutrition and UTI risk}

Interestingly, some of the reviewed studies also highlighted the influence of the severity/grades of malnutrition on the risk of UTI. ${ }^{7,14,17}$ In one of the studies in India, the authors reported that the risk of bacteriuria increased significantly with the severity of malnutrition and in patients with diarrhea. ${ }^{7}$ Their observation, however, contrasts with the findings of other studies in Nigeria ${ }^{14}$ and South Africa, ${ }^{17}$ which showed that prevalence rates among the grades of malnutrition did not vary significantly. The plausible explanation for the differences, however, remains unclear; one possible reason might be due to the differences in the methodologies followed in different studies: one was a case-control study, ${ }^{7}$ while the others were prospective studies with no controls. ${ }^{14,17}$

\section{Routine screening for UTI in children with SAM: is it an acceptable guideline?}

Current WHO guidelines recommend administering routine antibiotics for all children with SAM, even if they present with uncomplicated disease with no clinically evident infections including UTI. ${ }^{12}$ However, a recent systematic review and meta-analysis of three randomized controlled trials (RCTs), five Cochrane reviews, and 37 observational studies found little evidence underpinning the current WHO recommendations for routine antibiotics in uncomplicated SAM. ${ }^{8}$ The authors also observed that susceptibility studies favored amoxicillin over co-trimoxazole.

Guidelines or management protocols are predicated upon findings from studies with high levels of evidence. Unfortunately, there have been no RCTs on the diagnostic strategies or different treatments for UTI in children with SAM. ${ }^{22}$ However, with the relatively high prevalence of UTI reported among 
children with complicated SAM, it has been suggested that it would seem prudent to test and treat all children admitted with complicated SAM using urine dipstick or microscopy. ${ }^{21}$ The dipstick urinalysis essentially utilizes the leukocyte esterase and nitrite tests to establish the diagnosis of UTI. A positive leukocyte esterase test indicates the presence of pyuria, but a urine microscopy is required to confirm the finding. Pyuria is defined as the presence of $>10 \times 10^{6}$ white blood cells $(\mathrm{WBC}) / \mathrm{L}$ in an uncentrifuged urine sample or $>5 \times 10^{6} \mathrm{WBC} / \mathrm{L}$ in a centrifuged urine sample. ${ }^{37}$ The predictive value of pyuria as an isolated result is, however, poor and cannot be recommended for making a presumptive diagnosis of UTI, since it can also occur in other conditions such as febrile states of non-UTI origin, urolithiasis, and renal tuberculosis (the so-called "sterile pyuria"). ${ }^{5}$ The clinician is thus faced with two vital decisions before the treatment: administering empirical antibiotics for UTI or performing routine urine tests for UTI. A closer look at one of the globally accepted guidelines (National Institute for Health and Care Excellence [NICE] guidelines), may provide the clue to resolve this management dilemma. The NICE guidelines, published in 2007, have substantially changed clinical practice regarding the diagnosis, clinical management, and subsequent radiological investigation of the child with a UTI. ${ }^{38}$ The guidelines gave a summary of the treatment decisions to be taken depending on the results of the urine dipstick tests as follows: if leukocyte esterase and nitrite positive, start antibiotic treatment for UTI, and send urine for culture if high or intermediate risk of serious illness or past history of UTI; if leukocyte esterase negative and nitrite positive, start antibiotic treatment and send urine for culture; if leukocyte esterase positive and nitrite negative, treat only if there is a good clinical evidence of UTI, and culture the urine and treat depending on the result; and finally, if both leukocyte esterase and nitrite negative, do not treat as UTI or send urine for culture (Table 2). ${ }^{38}$ Although the guidelines were not necessarily formulated for UTI in children with SAM, they could equally be applicable in these children. Based on global reports, which indicate the preponderance of Gram-negative organisms as isolates, the choice of ampicillin and gentamicin as the first-line recommended broad spectrum antibiotics for complicated SAM should be able to cover the commonest community-acquired organisms causing UTI, the caveat being the absence of a high level of local resistance to gentamicin. Some authors recommend that urine tests should only be performed if a febrile illness in a child presenting with SAM fails to resolve after 48 hours of intravenous antibiotics. ${ }^{22}$ Thus, the need to perform routine urine tests as the first clinical decision at admission depends partly on the often unknown local-resistance patterns and partly on resources. Incidentally, the NICE guidelines seek to reduce the heavy burden on
Table 2 Guidelines on interpretation of urine dipstick results and management options for UTI in children

\begin{tabular}{ll}
\hline Dipstick urinalysis results & Recommended plan of action \\
\hline $\begin{array}{l}\text { Positive leukocyte esterase } \\
\text { and nitrite tests }\end{array}$ & $\begin{array}{l}\text { Antibiotic treatment for UTI } \\
\text { Urine culture if risk of serious illness is high } \\
\text { or intermediate and past history of UTI }\end{array}$ \\
Negative leukocyte esterase & $\begin{array}{l}\text { Antibiotic treatment } \\
\text { and positive nitrite tests }\end{array}$ \\
$\begin{array}{l}\text { Urine culture } \\
\text { Positive leukocyte esterase }\end{array}$ & $\begin{array}{l}\text { Antibiotic treatment only with good } \\
\text { clinical evidence of UTI }\end{array}$ \\
and negative nitrite tests & $\begin{array}{l}\text { Urine culture and treatment based on } \\
\text { results }\end{array}$ \\
Negative leukocyte and & $\begin{array}{l}\text { Antibiotic treatment and urine culture } \\
\text { not necessary }\end{array}$ \\
\hline
\end{tabular}

Note: National Institute for Health and Care Excellence (2007) adapted from CG54 Urinary tract infection in under 16s: Diagnosis and management. Manchester: NICE. Available from https://www.nice.org.uk/guidance/cg54. ${ }^{38}$ The material was accurate at the time of going to press.

Abbreviations: UTI, urinary tract infection; NICE, National Institute for Health and Care Excellence.

primary and secondary care resources, limit treatment costs, and promote evidence-based approach in the management of UTI in children. ${ }^{37}$ Despite the fact that the ability to perform urine culture and sensitivity test provides the possibility of tailoring treatment individually, the test may not be readily available in resource-poor countries where SAM is prevalent. Moreover, the test may not reveal all the bacterial pathogens involved. Therefore, in places of proven resistance to gentamicin, other antibiotics such as cephalosporin, nitrofurantoin, and ciprofloxacin have been suggested as reliable empirical alternatives. ${ }^{22}$

\section{Additional evaluation for UTI in children with SAM}

For children aged 6 months to 5 years, no further investigations are recommended for a UTI which responds well to treatment. ${ }^{22}$ Children with recurrent or persistent UTI require further evaluation to identify possible underlying anatomical abnormalities and sites of renal parenchymal damage or persistent infection. However, the current NICE guidelines recommend that different investigations should be performed according to whether there is a good response to treatment within 48 hours, whether there is evidence of atypical UTI, and whether there is evidence of recurrent UTI, and the age of the child should also be taken into consideration. ${ }^{38}$ While the estimation of plasma urea or creatinine is vital to establish renal impairment, additional radiological evaluation is performed based on the availability of investigations and resources. However, abdominal ultrasound scan to detect structural abnormalities remains essential and is fortunately accessible. Where resources are available, a micturating cystourethrogram and/or a dimercaptosuccinic acid scan at a 
tertiary facility should be the next line of investigation. These structural abnormalities may indeed serve as confounding variables in children with SAM whose immune deficiency and possible comorbid abnormalities constitute potential risk factors for recurrent UTI.

\section{Conclusion}

From global reports, there is overwhelming evidence to suggest that children with SAM have increased risk of UTI, although prevalence rates range from as low as $6 \%$ to as high as $37 \%$. The wide variation in prevalence rates could be explained by the use of different study methods, as well as consideration or nonconsideration of study confounders. The most common bacterial isolates from urine cultures remain Gram-negative coliform organisms such as E. coli and Klebsiella species. These findings have formed the basis for the current diagnostic and therapeutic guidelines for clinicians managing children with complicated SAM.

Despite the dearth of published studies on UTI among children with uncomplicated SAM, the existing data do not suggest a need to test every child with uncomplicated SAM for UTI. ${ }^{22}$ NICE guidelines have, however, been published as evidence-based recommendations to guide the clinician in the management algorithm of children with UTI. Among outpatients, performing routine dipstick urine test in febrile children with uncomplicated SAM may be an acceptable recommendation. Finally, given the high prevalence of UTI among children with complicated SAM, as reported in the literature, and concerns over antibiotic resistance, more extensive data using standardized microbiological methods are required. Thus, the assessment of the performance of urine dipsticks and microscopy against urine culture (which remains the gold standard) is an important step toward strengthening the evidence for the therapeutic guidelines for UTI in these children.

\section{Acknowledgment}

The author wishes to acknowledge the invaluable information obtained from the article by KDJ Jones and JA Berkley. ${ }^{22}$

\section{Disclosure}

The author reports no conflicts of interest in this work.

\section{References}

1. Hoberman A, Chao HP, Keller DM, Hickey R, Davis HW, Ellis D. Prevalence of urinary tract infection in febrile infants. JPediatr. 1993;123(1):17.

2. Shaw KN, Gorelick M, McGowan KL, Yakscoe NM, Schwartz JS. Prevalence of urinary tract infection in febrile young children in the emergency department. Pediatrics. 1998;102(2):e16.

3. Shaikh N, Morone NE, Bost JE, Farrell MH. Prevalence of urinary tract infection in childhood: a meta-analysis. Pediatr Infect Dis $J$. 2008;27(4):302.
4. Chang SL, Shortliffe LD. Pediatric urinary tract infections. Pediatr Clin North Am. 2006;53(3):379-400.

5. Srivastava RN, Bagga A. Urinary tract infection. In: Srivastava RN, Bagga A, editors. Pediatric Nephrology. 4th ed. New Delhi: Jaypee; 2005:235-264.

6. Roberts JA. Factors predisposing to urinary tract infections in children. Pediatr Nephrol. 1996;10(4):517-522.

7. Bagga A, Tripathi $\mathrm{P}$, Jatana $\mathrm{V}$, et al. Bacteriuria and urinary tract infections in malnourished children. Pediatr Nephrol. 2003;18(4): 366-370.

8. Alcoba G, Kerac M, Breysse S, et al. Do children with uncomplicated severe acute malnutrition need antibiotics? A systematic review and meta-analysis. PLoS ONE. 2013;8(1):e53184.

9. Iyer SS, Chatraw JH, Tan WG, et al. Protein energy malnutrition impairs homeostatic proliferation of memory CD8 T cells. J Immunol. 2012;188(1):77-84.

10. Babirekere-Iriso E, Musoke P, Kekitiinwa A. Bacteremia in severely malnourished children in an HIV-endemic setting. Ann Trop Paediatr. 2006;26(4):319-328.

11. WHO-UNICEF. WHO Child Growth Standards and the Identification of Severe Acute Malnutrition in Infants and Children: A Joint Statement WHO-UNICEF. 2009. Available from: http://www.who.int/nutrition/ publications/severemal nutrition/9789241598163_eng.pdf. Accessed December 5, 2012.

12. WHO. Management of Severe Malnutrition: A Manual for Physicians and Other Senior Health Workers. Geneva: World Health Organization; 1999. Available from: http://www.who.int/nutrition/publications/ malnutrition/en/index.html. Accessed July 2, 2012.

13. Prudhon C, Prinzo ZW, Briend A, Daelmans BM, Mason JB. Proceedings of the WHO, UNICEF, and SCN informal consultation on community-based management of severe malnutrition in children. Food Nutr Bull. 2006;27(3):S99-104.

14. Rabasa AI, Shattima D. Urinary tract infection in severely malnourished children at the University of Maiduguri Teaching Hospital. J Trop Pediatr. 2002;48(6):359-361.

15. Page A-L, de Rekeneire N, Sayadi S, et al. Infections in children admitted with complicated severe acute malnutrition in Niger. PLOS ONE. 2013;8(7):e68699.

16. Kala UK, Jacobs DW. Evaluation of urinary tract infection in malnourished black children. Ann Trop Paediatr. 1992;12(1):75-81.

17. Reed RP, Wegerhoff FO. Urinary tract infection in malnourished rural African children. Ann Trop Paediatr. 1995;15(1):21-26.

18. Berkowitz FE. Infections in children with severe protein-energy malnutrition. Ann Trop Paediatr. 1983;3(2):79-83.

19. Shisana O, Rehle T, Simbayi LC, et al. South African National HIV Prevalence, Incidence and Behaviour Survey, 2012. Cape Town, South Africa: HSRC Press; 2014

20. Shimeles D, Lulseged S. Clinical profile and pattern of infection in Ethiopian children with severe protein-energy malnutrition. East Afr Med J. 1994;71(4):264-267.

21. Thuo N, Ohuma E, Karisa J, Talbert A, Berkley JA, Maitland K. The prognostic value of dipstick urinalysis in children admitted to hospital with severe malnutrition. Arch Dis Child. 2010;95(6):422-426.

22. Jones KDJ, Berkley JA. Severe acute malnutrition and infection. Paediatr Int Child Health. 2014;34(S1):S1-S29.

23. Caksen H, Cesur Y, Uner A, et al. Urinary tract infection and antibiotic susceptibility in malnourished children. Int J Urol Nephrol. 2000;32(2):245-247.

24. James-Ellison MY, Roberts R, Verrier-Jones K, Williams JD, Topley N. Mucosal immunity in the urinary tract: changes in sIgA, FSC and total IgA with age and in urinary tract infection. Clin Nephrol. 1997;48(2):69-78.

25. Deo SS, Vaidya AK. Elevated levels of secretory immunoglobulin A (sIgA) in urinary tract infections. Indian J Pediatr. 2004;71(1):37-40.

26. Fliedner M, Mehls O, Rauterberg EW, Ritz E. Urinary sIgA in children with urinary tract infection. J Pediatr. 1986;109(3):416-421.

27. Riedasch G, Heck P, Rauterberg E, Ritz E. Does low urinary sIgA predispose to urinary tract infection? Kidney Int. 1983;23(5):759-763. 
28. McGee DW, McMurray DN. The effect of protein malnutrition on the IgA immune response in mice. Immunology. 1988;63(1):25-29.

29. McGee DW, McMurray DN. Protein malnutrition reduces the IgA immune response to oral antigen by altering B-cell and suppressor T-cell functions. Immunology. 1988;64(4):697-702.

30. Sullivan DA, Vaerman JP, Soo C. Influence of severe protein malnutrition on rat lacrimal, salivary and gastrointestinal immune expression during development, adulthood and ageing. Immunology. 1993;78(2):308-317.

31. Asharam K, Bhimma R, Adhikari M. Hum an immunodeficiency virus and urinary tract infections in children. Ann Trop Paediatr. 2003; 23(4):273-277.

32. Laway MA, Wani ML, Patnaik R, et al. Does circumcision alter the periurethral uropathogenic bacterial flora? Afr J Paediatr Surg. 2012;9(2):109-112.

33. Banapurmath CR, Jayamony S. Prevalence of urinary tract infection in severely malnourished preschool children. Indian Pediatr. 1994;31(6):679-682.
34. Ahmed M, Moremi N, Mirambo MM, et al. Multi-drug gram-negative enteric bacteria causing urinary tract infection among malnourished under-fives admitted at a tertiary hospital, northwestern Tanzania. Ital J Pediatr. 2015;41:44.

35. Okomo UA, Garba D, Fombah AE, et al. Bacterial isolates and antibiotic sensitivity among Gambian children with severe acute malnutrition. Int J Pediatr. 2011;2011:825123.

36. Brown KH, Gilman RH, Gaffar A, et al. Infections associated with severe protein-calorie malnutrition in hospitalized infants and children Nutr Res. 1981;1(1):33-46.

37. Rees L. Urinary tract infection. In: Rees L, Brogan PA, Bockenhauer D, Webb NJA, editors. Paediatric Nephrology-Oxford Specialist Handbooks in Paediatrics. 2nd ed. Oxford University Press; 2012.

38. National Institute for Health and Clinical Excellence. Urinary Tract Infection in Children. NICE Clinical Guideline 54. London: NICE; 2007.
Pediatric Health, Medicine and Therapeutics

\section{Publish your work in this journal}

Pediatric Health, Medicine and Therapeutics is an international, peerreviewed, open access journal publishing original research, reports, editorials, reviews and commentaries. All aspects of health maintenance, preventative measures and disease treatment interventions are addressed within the journal. Practitioners from all disciplines are invited to submit

\section{Dovepress}

their work as well as healthcare researchers and patient support groups. The manuscript management system is completely online and includes a very quick and fair peer-review system. Visit http://www.dovepress.com/ testimonials.php to read real quotes from published authors. 